Published in final edited form as:

Res Social Adm Pharm. 2019 June ; 15(6): 801-805. doi:10.1016/j.sapharm.2018.08.013.

\title{
Deprescribing: future directions for research
}

\author{
Wade Thompson, BScPhm, PharmD, MSc ${ }^{\mathrm{a},{ }^{,} \text {, }}$
}

Research Unit of General Practice, Department of Public Health, University of Southern Denmark, JB Winsløwsvej 9A, Odense, Denmark, 5000C

Emily Reeve, BPharm(Hons), $\mathrm{PhD}^{\mathrm{b}, \mathrm{c}}$,

Aging and Pharmacology, L13 Kolling Institute, Royal North Shore Hospital, St Leonards, NSW, 2065, Australia

Frank Moriarty, BScPharm, MPharm, PhD $^{d}$,

HRB Centre for Primary Health Care Research, Royal College of Surgeons in Ireland, $123 \mathrm{St}$ Stephen's Green, Dublin, D02 YN77, Ireland

Malcolm Maclure, ScDe,

Department of Anesthesiology, Pharmacology and Therapeutics, University of British Columbia, 2775 Laurel Street, Vancouver BC, V5Z 1M9

Justin Turner, BPharm, MClinPharm, PhD $^{f}$,

Faculty of Pharmacy, University of Montreal, Centre de recherche, Institut Universitaire de Gériatrie de Montréal, 4545 Chemin Queen Mary, Montréal, Canada, H3W 1W4

Michael A. Steinman, MDg,

Division of Geriatrics, Department of Medicine, University of California San Francisco, 4150 Clement St, Box 181G, San Francisco, USA, 94121

James Conklin, $\mathrm{PhD}^{\mathrm{h}, \mathrm{i}}$,

\footnotetext{
*Corresponding author: wthomp01@gmail.com, Phone: +45 2077 9527, Address: Winsløwsvej 9A, Odense, Denmark, 5000C. *** Symposium lead

Conflicts of interest

Dr. Thompson reports honoraria for deprescribing presentations from University of Ottawa , University of British Columbia and Ontario Long-Term Care Clinicians Conference. Dr. Reeve reports grants from Australian National Health and Medical Research Council (NHMRC)-Australian Research Council (ARC), a research award from the Bupa Health Foundation and deprescribing presentation fees from the Pharmacy Association of Nova Scotia and the Swiss Society of Internal Medicine. Dr. McCarthy reports travel support for a presentation from NHS England. Dr. Farrell reports honoraria for deprescribing presentations from College of Psychiatric and Neurologic Pharmacists, European Association of Hospital Pharmacists, Nova Scotia College of Pharmacists. Drs. Farrell and Conklin received a stipend from the Institute for Healthcare Improvement for advice and review of documents related to introducing deprescribing into the US healthcare system.
} 
Bruyère Research Institute, 43 Bruyère St., Ottawa, Ontario, K1N 5C8

Lisa Dolovich, BScPhm, PharmD, MScj,

Leslie Dan Faculty of Pharmacy, University of Toronto, 144 College Street, Toronto, Ontario M5S $3 \mathrm{M} 2$

\section{Lisa McCarthy, BScPhm, PharmD, MSc ${ }^{\mathrm{j}, \mathrm{k}}$, and}

Women's College Research Institute, 76 Grenville St., Toronto, Ontario, M5S 1B2

Barbara Farrell, BScPhm, PharmD ${ }^{h, l, m,{ }^{* *}}$

Bruyère Research Institute, 43 Bruyère St., Ottawa, Ontario, K1N 5C8

aResearch Unit of General Practice, Department of Public Health, University of Southern Denmark, Odense, Denmark ${ }^{b}$ HHMRC Cognitive Decline Partnership Centre, Kolling Institute of Medical Research, Northern Clinical School, Faculty of Medicine and Health, University of Sydney, Sydney, Australia, 'Geriatric Medicine Research, Queen Elizabeth II Health Sciences Centre, Halifax, Canada dHRB Centre for Primary Care Research, Royal College of Surgeons in Ireland, Dublin, Ireland eDepartment of Anesthesiology, Pharmacology and Therapeutics, University of British Columbia, Vancouver, British Columbia fFaculty of Pharmacy, University of Montreal, Centre de recherche, Institut Universitaire de Gériatrie de Montréal, Montréal, Canada, gDivision of Geriatrics, Department of Medicine, University of California San Francisco, San Francisco, USA hBruyère Research Institute, Ottawa, Ontario, Canada 'Concordia University, Montreal, Quebec, Canada 'Leslie Dan Faculty of Pharmacy, University of Toronto, Toronto, Canada kWomen's College Research Institute, Women's College Hospital, Toronto, Canada 'Department of Family Medicine, University of Ottawa, Ottawa, Ontario, Canada mSchool of Pharmacy, University of Waterloo, Kitchener, Ontario, Canada

\section{Abstract}

A World Café workshop was held at the Bruyère Evidence-Based Deprescribing Guidelines Symposium in March 2018 with 30 participants (researchers, clinicians, policy makers, stakeholders). This workshop explored priorities for future work in the field of deprescribing and deprescribing guidelines through group discussion. The discussions were guided by the following questions: (1) What are deprescribing research priorities (to inform guideline development), (2) What outcome measures are important for developing deprescribing guidelines, and (3) How do we evaluate the implementation and effectiveness of deprescribing guidelines? Discussion from all 3 questions identified 6 main priority areas: (1) conducting high-quality and long-term clinical trials that measure patient-important outcomes, (2) focusing on patient involvement and perspectives, (3) investigating the pharmacoeconomics of deprescribing interventions, (4) understanding deprescribing interventions in different populations, (5) generating evidence on clinical management during deprescribing (e.g. managing adverse drug withdrawal effects, subsequent re-prescribing), and (6) implementing interventions in clinical practice. These topics 
represent what a group of experienced researchers, clinicians, and stakeholders in the field collectively felt was important to consider for design and implementation of future deprescribing studies. The aim for these findings is to stimulate future discussions and be considered by granting agencies, policy makers, deprescribing research networks, and individual researchers planning future deprescribing studies.

\section{Keywords}

deprescribing; guidelines; polypharmacy; research

\section{Introduction}

The Bruyère Evidence-Based Deprescribing Guidelines Symposium took place in Ottawa, Canada from March 27-29, 2018. This symposium brought together researchers, educators, clinicians, patient advocates, guideline developers, policy makers, and other stakeholders. A major goal was to identify future research directions that build on previous deprescribing research. The symposium included a workshop where participants met to discuss gaps in the deprescribing evidence base and directions for future deprescribing research.

There has been an exponential increase in deprescribing research publications in recent years. ${ }^{1-4}$ These include studies of medication reviews or educational interventions to facilitate deprescribing behaviour, as well as targeted medication withdrawal studies and some economic evaluations. ${ }^{3}$ While such interventions have been shown to be effective (particularly for reductions in medication use) and safe, their generalizability is limited due to small sample sizes, lack of control groups, and short-term follow-up. Studies of patient attitudes and preferences for deprescribing have been published, ${ }^{5,6}$ but there has been little investigation into how to operationalize these findings in practice.

The Bruyère Deprescribing Guidelines research team has used a rigorous, systematic approach to develop deprescribing guidelines and decision-support tools. ${ }^{7}$ Many are using these tools and have asked for guidance on evaluating their implementation and effectiveness. The work in developing rigorous and high-quality deprescribing guidelines to date has made it clear that there are still limitations and gaps in the evidence required to create new guidelines and to evaluate their effectiveness. Addressing these gaps will enhance development of further high-quality evidence-based deprescribing guidelines and other quality deprescribing tools and interventions.

The research workshop at the symposium aimed to identify areas where evidence is most urgently needed and discuss how to best address these gaps and limitations in evidence. This article describes the dialogue and findings that came out of this workshop. The aim of sharing the findings is to encourage further conversations about how to efficiently continue research into deprescribing. 


\section{Design}

The research workshop was conducted according to the principles of a World Café. This is a dialogue method to facilitate group discussion and summarize the viewpoints of large groups. ${ }^{8,9}$ It is based on the principle that the best ideas are generated through open-ended discussions rather than through a more directive framework (e.g. structured interviews or focus groups).

Participants (approximately 30) who conduct or are interested in conducting research in the field of deprescribing and/or polypharmacy took part. They were first presented with an overview of participatory and expert-led methodologies and approaches that could be used to evaluate deprescribing initiatives as well as an overview of deprescribing research to date and gaps in the deprescribing evidence base. Slides from these presentations can be accessed here. Three questions were developed by the workshop organizers (ER, WT) based on their experiences developing deprescribing guidelines and conducting deprescribing research (see Box 1). The first 2 discussions posed questions aimed at identifying research priorities to inform new deprescribing guideline development and identify important outcome measures for studies to inform guideline development. Following lunch, participants were invited to deliver 2-minute rapid-fire presentations of their deprescribing research. The intention was to give researchers an opportunity to share their work, as well as to stimulate ideas for discussion. The third World Café discussion then explored methods for evaluating the implementation and effectiveness of deprescribing guidelines. A brief outline of the process and discussion questions is provided in Box 1.

The first question aimed to identify deprescribing research priorities needed to inform future guideline development. This question was chosen because many priorities for deprescribing guidelines have been identified ${ }^{10}$ and lists of potentially inappropriate medications are widely available. Yet literature reviews undertaken by our research team have revealed a lack of adequately powered controlled deprescribing trials that consider patient-important outcomes upon which to build evidence-based guidelines for deprescribing. The second question sought to generate important patient outcomes which should be used in deprescribing studies (with a focus on the purpose of developing deprescribing guidelines). Finally, the third question was intended to stimulate discussion of methods and approaches for evaluating implementation of deprescribing guideline initiatives moving forward. While the questions focused on research related to development and implementation of evidencebased deprescribing guidelines, discussion could also consider the wider field of deprescribing research.

\section{Findings}

\section{Deprescribing research priorities: research to inform future deprescribing guideline development}

The questions about (1) deprescribing research priorities to inform future deprescribing guideline development and (2) important outcome measures for future deprescribing studies yielded priorities in relation to 6 categories. Findings are summarized in Figure 1. 


\section{Deprescribing trials (design and clinical outcome measurement)-}

Participants identified a need to conduct informative, high-quality clinical trials of broad patient-centered deprescribing interventions, as well as trials of targeted drug-specific medication withdrawal. It was noted that both types of trials, broad patient-centred deprescribing and deprescribing of specific drug classes, could include the measurement of clinically relevant outcomes. For example, studies could measure the clinical and patientimportant outcomes of deprescribing rather than only using numbers of medications deprescribed (or rate of medication discontinuation) as a primary outcome; these would include measurement of quality of life, potential harms from deprescribing (e.g. adverse drug withdrawal events [ADWEs])- adverse effects caused by discontinuation of a medication) and potential reductions in adverse drug events such as falls, cognitive impairment, reduced physical function, and mortality. Participants also brought forward the idea that there could be an increasing focus on "no change" in clinical status as an outcome in deprescribing trials. That is, if there was no change (e.g. in patients' symptoms or function) following deprescribing, that could be viewed as a positive outcome given a potential reduction in pill burden and costs. Non-inferiority designs could help evaluate whether deprescribing results in "no change" in clinical status.

Trials could also be adequately powered to detect a difference or equivalence in the primary outcome. While it may not always be viable for individual randomized controlled trials (RCTs) to recruit enough patients to detect a meaningful difference in certain rarer clinical outcomes (e.g. mortality), routine and consistent measurement of clinical outcomes would create the possibility of future meta-analyses. The newly developed core outcome set for clinical trials of interventions to manage polypharmacy may be helpful to facilitate this; however, specific tools to measure these outcomes have not yet been decided upon, and studies of deprescribing may pose unique considerations that require special approaches to outcomes and measurement. ${ }^{11}$ As conducting sufficiently powered, long-term RCTs are likely to be infeasible for all populations and drug classes, deprescribing research can also utilize pharmacoepidemiological methods (e.g. observational studies of existing clinical cohorts) and pragmatic trials. ${ }^{12,13}$

Participants also highlighted the need to measure downstream effects of deprescribing. These include: re-prescribing of the original medication, substitution with an alternate medication, or starting over-the-counter medications. This could increase understanding of the sustainability and the long-term clinical impact of deprescribing.

2. Engaging patients and focusing on patient perspectives-The centrality of patients (and family members/caregivers) to deprescribing was discussed throughout, for example highlighting the need for identifying and measuring outcomes important to patients. There was specific discussion about increasing patient education, engagement and empowerment, and shared decision-making. The group felt that research into how to best make patients aware of deprescribing as a normal and positive part of care was deemed important for optimizing deprescribing at the point of care. Improving understanding of how to best achieve shared decision-making about deprescribing was similarly highlighted as an important area to address for future work. 
The patient, caregiver, and family member experience was felt to be an important outcome to measure in deprescribing studies. This could be evaluated using patient/caregiver satisfaction measures (for example, measuring patient satisfaction with the deprescribing intervention as an outcome). Studies could also examine whether, and how, care from deprescribing interventions (e.g. changes in drug therapy plans) aligns with patient goals, values, and preferences. Participants mentioned that it would be helpful to measure patient self-efficacy in the deprescribing process and identify methods to improve self-efficacy.

3. Pharmacoeconomic research-Research into the cost-effectiveness of deprescribing was highlighted as a priority. The group noted that deprescribing interventions can be time-consuming and resource-intensive and therefore robust data on the impact of interventions on health services utilization and health care costs, costs of the intervention, and cost-effectiveness is required for effecting sustainable change in policy and practice.

Several of the outcome measures discussed above result in high health care system costs. Therefore, possible reductions in emergency room visits, falls, hospitalizations, and drug costs, and improvements in quality of life may generate health-system savings that need to be balanced against the cost associated with implementing deprescribing initiatives (e.g. clinician time, resources) as well as managing ADWEs. Pharmacoeconomic studies of deprescribing should encompass patient, health system, and societal perspectives to ensure evaluation on how deprescribing in one care environment may impact the cost and resource use in other care environments (e.g. deprescribing in hospital to reduce burden of care in primary care). Measurement of clinical outcomes and quality-of-life would provide insight into potential model inputs and would facilitate the conduct of cost-effectiveness and costutility modelling and to understand circumstances in which deprescribing is most costeffective.

The cost of non-adherence was also discussed as it represents a major economic burden on the health care system. ${ }^{14}$ It is possible that deprescribing unnecessary medications may lead to improvements in adherence for necessary medications. ${ }^{15}$ Such improvements in adherence from deprescribing could potentially save money spent on negative outcomes of non-adherence, or on medications that were not being consumed.

4. Target populations-The effects of deprescribing interventions in different patient populations were seen as important. Specifically, evaluations could focus on how to identify patient groups that are likely to derive the greatest benefit from deprescribing interventions and examine how an intervention impacts different patient groups. Research could examine optimal mechanisms to prioritize patients to receive targeted deprescribing interventions in practice (e.g. how to best identify "high-risk" patients to receive deprescribing interventions). Leveraging existing literature could be important here. For example, to understand the effect of deprescribing in different populations (and identify "target" groups) synthesizing existing literature on harms of polypharmacy may be informative. We could synthesize existing literature on deprescribing to more closely investigate the effects in different patient groups/settings or use existing systematic reviews/meta-analyses of deprescribing to identify patient groups most likely to benefit. Participants also suggested that while older persons are at high risk of harm from polypharmacy, it may be important to 
examine whether deprescribing interventions in younger patients could avoid downstream harm from polypharmacy.

5. Clinical management during deprescribing-Participants noted that more research is needed on optimal clinical management of patients during the deprescribing process. One such area is management of ADWES. For example, research could focus on what to monitor, how often to monitor, and what to do if ADWEs occur. While awareness of ADWEs for some categories of medications exists, the group felt that more research is needed to understand how to best manage these events (e.g. alternative medications, frequency of follow-up, watching and waiting). This would give practicing clinicians useful, evidence-based advice to implement in clinical practice. Effectiveness and implementation of non-pharmacological measures following deprescribing and avoidance of substitution with other potentially harmful medications is another potential area of study.

6. Implementation-Participants felt future research should use an implementation science lens to examine what works, for whom, and why. These approaches would allow researchers to identify how deprescribing interventions can be best delivered in different practice settings. For example, we could learn more about how to tailor deprescribing interventions in different settings (e.g. primary care versus long-term care). The group also felt studies should consider the different health care levels such as patients/public, clinicians, health care organizations and the wider environment (policy, regulation, etc.). This might involve interventions which target these different levels or investigation into how deprescribing can be facilitated at these different levels (i.e. review of existing processes and tasks). For example, studies could explore whether different policy initiatives are effective at increasing deprescribing activities. Lastly, studies need to evaluate how interventions are sustained over time and whether there are mechanisms that may improve sustainability.

The group also suggested further research into deprescribing roles. It was acknowledged that deprescribing is a team effort. However, it would be helpful to understand how each individual health care provider (e.g. specialist, family/primary care doctor, pharmacist, nurse) could contribute to the deprescribing process (i.e. who does what best?). Studies which compare different models and role responsibilities would help us understand how to operationalize teams to implement deprescribing strategies. Further, studies could evaluate the health care provider experience with implementing or using a deprescribing intervention in clinical practice to identify barriers and facilitators to uptake of specific deprescribing interventions. They could also evaluate experience at different levels of interventions (e.g. patient-centred interventions, education interventions). These studies would also contribute to understanding of deprescribing roles and how to best increase deprescribing in practice.

\section{Evaluating the implementation and effectiveness of deprescribing guidelines}

The third and final question concerned optimal ways to evaluate the implementation and effectiveness of deprescribing guidelines. Answers to this question largely reiterated the themes noted above, though participants also raised novel points. They agreed that evaluation of guideline implementation should involve a multi-methods approach overall, incorporating both quantitative and qualitative measurements. For example, a study might 
conduct surveys and interviews on patient and clinician experience along with quantitative measures of effectiveness. It was felt that carefully selected control or comparator groups would also be needed to accurately compare implementation of a deprescribing guideline with usual care. Existing implementation and evaluation frameworks could be used to guide study design. ${ }^{16}$

\section{Implications for future studies}

This paper presents several areas that researchers and clinicians working in the field of deprescribing felt were important to address in future research. The priorities for future research discussed during this workshop mirrored many of the known gaps in evidence discussed in current literature. ${ }^{3,17}$ Regardless of the study design or patient population, the group identified the need for sharing and collaboration amongst the international deprescribing community. This could take the form of a clinical trials network for deprescribing or special interest groups for deprescribing in specific disease states. It was felt that such initiatives would reduce duplication of effort, foster collaborations, and increase capacity to conduct deprescribing studies.

\section{Strengths and weaknesses}

There was a diverse group of participants. This allowed for differing and unique perspectives during discussions to generate new ideas. However, no consensus was systematically sought on the ideas brought forward or prioritization of which topics or ideas were the most important. Rather, the workshop served as a focused brainstorming session and open forum for an exchange of ideas. Thus, the results represent what a group of experienced researchers, clinicians, and stakeholders in the field collectively felt was important to consider for design and implementation of future deprescribing studies. The aim is for the findings to stimulate future discussions and be considered by granting agencies, policy makers, deprescribing research networks, and individual researchers planning future deprescribing studies.

\section{Conclusions}

The world café workshop brought together a group of researchers and clinicians with experience and interest in deprescribing research. Several areas of focus for future deprescribing studies were identified. Future deprescribing research should improve understanding of the benefits and harms of deprescribing medication classes (focusing on clinical outcomes), provide guidance on how to best manage withdrawal effects, understand patient experience, promote shared decisions, and allow development, implementation, and evaluation of interventions that are feasible, sustainable, and cost-effective. The overall aim of such studies is to optimize deprescribing in practice, and as such improve patient care by minimizing medication-induced harm. 


\section{Acknowledgements}

Thank you to the many notetakers and to Salima Suleman for her work in compiling and summarizing the notes from each workshop table. The team also thanks Heera Sen and Stephen Smith for their assistance in preparing these commentaries for publication.

Funding Funding for the planning and implementation of the symposium was provided by a Knowledge Mobilization Partnership Program grant through the Centre for Aging and Brain Health Innovation. Additional financial support was provided by the Bruyère Centre for Learning, Research and Innovation, the Canadian Foundation for Healthcare Improvement, CADTH, Medical Pharmacies and the Canadian Deprescribing Network. Funders were not involved in the writing of this report.

\section{References}

1. Iyer S, Naganathan V, Mclachlan AJ, Couteur DG Le. Medication Withdrawal Trials in People Aged 65 Years and Older A Systematic Review. Drugs Aging. 2008;25(12):1021-1031. [PubMed: 19021301]

2. Gnjidic D, Le Couteur DG, Kouladjian L, Hilmer SN. Deprescribing Trials: Methods to Reduce Polypharmacy and the Impact on Prescribing and Clinical Outcomes. Clin Geriatr Med. 2012;28(2): 237-253. doi:10.1016/j.cger.2012.01.006 [PubMed: 22500541]

3. Reeve E, Thompson W, Farrell B. Deprescribing: A narrative review of the evidence and practical recommendations for recognizing opportunities and taking action. Eur J Intern Med. 2017;38:3-11. doi:10.1016/j.ejim.2016.12.021 [PubMed: 28063660]

4. Page AT, Clifford RM, Potter K, Schwartz D, Etherton-Beer CD. The feasibility and effect of deprescribing in older adults on mortality and health: a systematic review and meta-analysis. Br J Clin Pharmacol. 2016;82(3):583-623. doi:10.1111/bcp.12975 [PubMed: 27077231]

5. Weir K, Nickel B, Naganathan V, et al. Decision-Making Preferences and Deprescribing: Perspectives of Older Adults and Companions About Their Medicines. Journals Gerontol Ser B. 2017;0(0):1-10. doi:10.1093/geronb/gbx138

6. Jansen J, Naganathan V, Carter SM, et al. Too much medicine in older people? Deprescribing through shared decision making. BMJ. 2016;353(June). doi:10.1136/bmj.i2893

7. Farrell B, Pottie K, Rojas-Fernandez CH, Bjerre LM, Thompson W, Welch V. Methodology for developing deprescribing guidelines: Using evidence and GRADE to guide recommendations for deprescribing. PLoS One. 2016;11(8). doi:10.1371/journal.pone.0161248

8. Estacio EV, Karic T. The World Café: An innovative method to facilitate reflections on internationalisation in higher education. J Furth High Educ. 2016;40(6):731-745. doi: 10.1080/0309877X.2015.1014315

9. Brown J Shaping Our Futures Through Conversations That Matter. San Francisco: Berrett-Koehler Publishers; 2005.

10. Farrell B, Tsang C, Raman-Wilms L, Irving H, Conklin J, Pottie KWhat Are Priorities for Deprescribing for Elderly Patients? Capturing the Voice of Practitioners: A Modified Delphi Process. Dalal K, ed. PLoS One. 2015;10(4):e0122246. doi:10.1371/journal.pone.0122246 [PubMed: 25849568]

11. Rankin A, Cadogan CA, Ryan C, Clyne B, Smith SM, Hughes CM. Core Outcome Set for Trials Aimed at Improving the Appropriateness of Polypharmacy in Older People in Primary Care. J Am Geriatr Soc. 2018. doi:10.1111/jgs. 15245

12. Peters M, Crocker H, Jenkinson C, Doll H, Fitzpatrick R. The routine collection of patient-reported outcome measures (PROMs) for long-term conditions in primary care: a cohort survey. BMJ Open. 2014;4(2):e003968. doi:10.1136/bmjopen-2013-003968

13. Staa T-P van, Goldacre B, Gulliford M, et al. Pragmatic randomised trials using routine electronic health records: putting them to the test. BMJ. 2012;344:e55. [PubMed: 22315246]

14. Cutler RL, Fernandez-Llimos F, Frommer M, Benrimoj C, Garcia-Cardenas V. Economic impact of medication non-adherence by disease groups: a systematic review. BMJ Open. 2018;8(1):e016982. doi:10.1136/bmjopen-2017-016982 
15. Reeve E, Wiese MD. Benefits of deprescribing on patients' adherence to medications. Int J Clin Pharm. 2014;36(1):26-29. doi:10.1007/s11096-013-9871-z [PubMed: 24242974]

16. Glasgow RE, Vogt TM, Boles SM. Evaluating the public health impact of health promotion interventions: the RE-AIM framework. Am J Public Health. 1999;89(9):1322-1327. [PubMed: 10474547]

17. Tan ECK, Sluggett JK, Johnell K, et al. Research Priorities for Optimizing Geriatric Pharmacotherapy: An International Consensus. J Am Med Dir Assoc. 2018;19(3):193-199. doi: 10.1016/j.jamda.2017.12.002 [PubMed: 29361432] 


\section{Box 1}

Participants sat at round tables, in groups of 4-6 with one person in the role of 'note-taker'.

"What are deprescribing research priorities (to inform guideline development)?" (30 minutes)

- Discussion round 1: Groups discussed question 1 at each table for 10 minutes, with the notetaker taking notes to summarize the discussion.

- After 10 minutes: the notetaker remained at their original table while the other participants moved to different tables.

- $\quad$ Discussion round 2: The notetaker presented the summary of the previous discussion to the newly formed group and then the group discussed Question 1 again for 10 minutes with the notetaker adding to their notes.

- Group presentation: Table by table, each notetaker provided a summary to the whole group and the entire group discussed the question.

This process was repeated for Questions 2 and 3:

"What outcome measures are important for developing deprescribing guidelines?" (30 minutes)

- $\quad$ Discussion round 1.

- After 10 minutes: notetaker remained at original table, other participants to different tables.

- $\quad$ Discussion round 2.

- $\quad$ Group presentation.

"How do we evaluate the implementation and effectiveness of deprescribing guidelines?"' (40 minutes)

- $\quad$ Discussion round 1.

- After 10 minutes: notetaker remained at original table, other participants to different tables.

- $\quad$ Discussion round 2.

- After 10 minutes: notetaker remained at original table, other participants to different tables.

- Discussion round 3.

- $\quad$ Group presentation. 


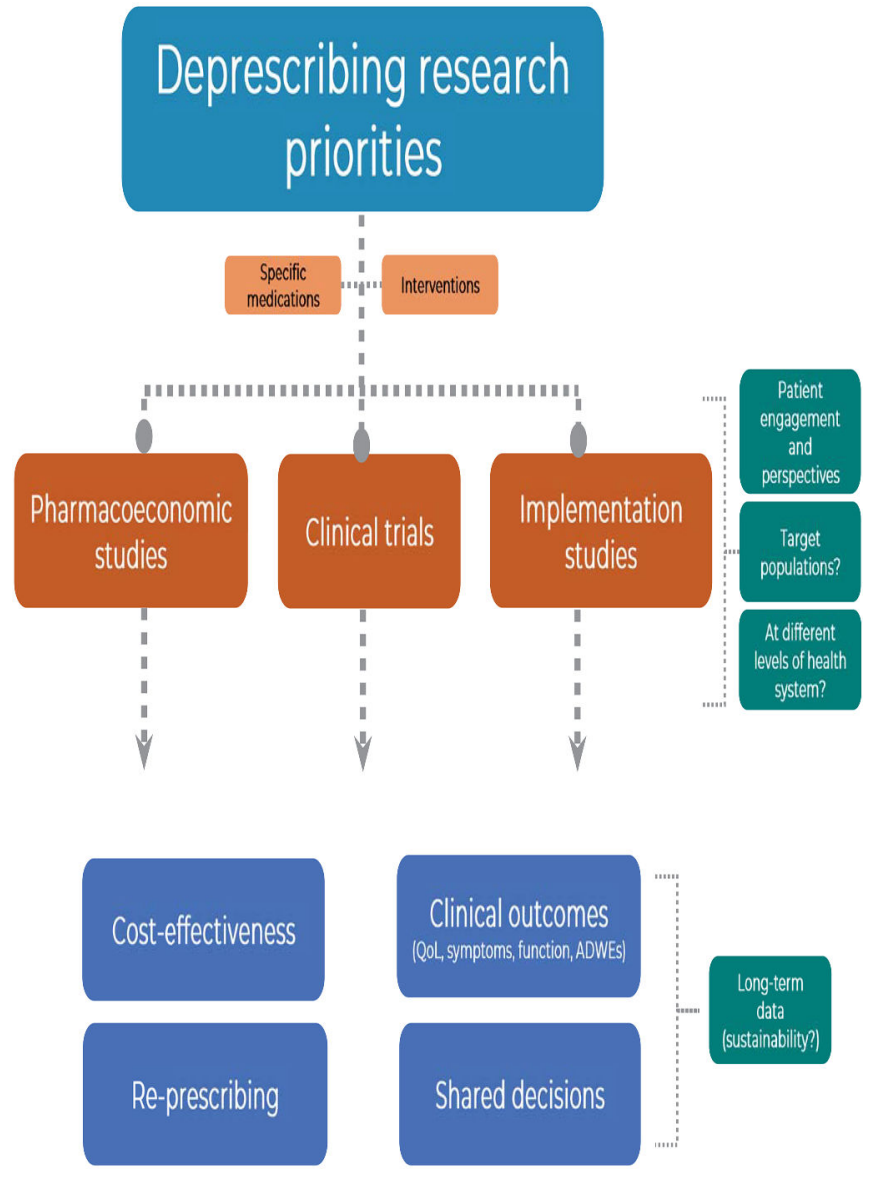

Fig 1.

Summary of deprescribing research priorities. 